\title{
La dieta rica en frutas y verduras y pobre en grasas disminuye la presión arterial en normotensos e hipertensos
}

A clinical trial of the effects of dietary patterns on blood pressure.

Appel LJ; Moore TJ; Obarzaneck E y col. (Grupo DASH: Dietary Approaches to Stop Hypertension). N Eng J Med 1997;336:117-24

\section{Objetivo}

Evaluar el efecto de la dieta rica en frutas y verduras, hipograsa o no, en los valores de la presión arterial.

\section{Diseño}

Ensayo multicéntrico randomizado y controlado. Ocho semanas de seguimiento.

\section{Lugar}

Estados Unidos.

\section{Pacientes}

Se incluyeron 459 personas mayores de 22 años con Tensión Arterial Sistólica (TAS) < 160, Diastólica (TAD) entre 80 y 95 y sin tratamiento antihipertensivo ni enfermedades crónicas como diabetes mal controlada, hiperlipidemia, obesidad y otras. El $60 \%$ eran de raza negra, y $49 \%$ eran mujeres.

\section{Intervención}

Grupo 1: dieta rica en frutas y verduras; Grupo 2: dieta combinada, igual a la anterior más lácteos descrema- dos y escasa cantidad de colesterol, de grasas en general y saturadas y Grupo 3: dieta promedio de los habitantes de EEUU. El peso y consumo de sodio fueron mantenidos estables.

\section{Medición de resultados principales}

Cambios en la TAD en reposo; y secundariamente la TAS y el promedio del holter de presión de 24 horas.

\section{Resultados principales}

La dieta rica en frutas y verduras redujo la TAS en 2,8 $\mathrm{mmHg}$ y la TAD en $1,9 \mathrm{mmHg}$ comparada con la dieta promedio y la dieta combinada redujo la TAS en $5,5 \mathrm{mmHg}$ y la TAD en $3,0 \mathrm{mmHg}$ comparada con la dieta promedio. Estas disminuciones fueron evidentes desde la segunda semana y se comprobaron tanto en normotensos como en hipertensos.

\section{Conclusiones}

Los resultados sugieren que tanto en normotensos como en hipertensos estadío 1 (leve) la dieta tiene un efecto hipotensor (en el corto plazo estudiado) similar al obtenido con fármacos.

Apoyo financiero: National Heart, Lung and Blood Institute; the Office of Research on Minority Health and the National Center for Research Resources of the National Institutes of Health.

\section{Comentario}

La dieta hiposódica; el límite al consumo del alcohol y al de las calorías en general; y el aumento del consumo de potasio están recomendadas para el tratamiento de la HTA como primera alternativa. No hay evidencia concluyente sobre el efecto de la dieta rica en frutas y verduras y pobre en grasas, sobre la presión arterial. Los estudios observacionales de dietas vegetarianas demostraron un efecto; sin embargo en los estudios experimentales que se basaron en suplementos específicos como fibra, potasio, magnesio, calcio y proteínas el efecto ha sido pequeño o inconsistente. Las posibles razones podrían ser que cada nutriente tiene un efecto pequeño que en los estudios observacionales se manifiestarían asociados; que otros nutrientes de estas die- tas, pero no los estudiados aisladamente, serían los responsables del efecto y que el efecto se presentaría cuando se los consume en los alimentos y no como suplementos.

Este estudio experimental demuestra el efecto general de la dieta de frutas y verduras y de la dieta combinada sobre la presión arterial en normotensos y en hipertensos estadío 1 (leve). No se pretendió identificar cuál o cuáles nutrientes específicos fueron los responsables del efecto. Las limitaciones de esta nueva evidencia son: a) no evalúa el efecto de las dietas a largo plazo, sobre el control de la presión y sobre los eventos cardiovasculares; b) la alta proporción de afro-americanos $(60 \%)$ no representa a nuestro país; c) hay que ser cuidadosos al extrapolar estos resultados de eficacia, en condiciones experi- mentales, a nuestra práctica ambulatoria, puesto que los participantes retiraban diariamente la vianda para facilitarles la adherencia. Sin embargo, la práctica médica obliga a tomar decisiones en el momento actual y usar el criterio para valorar la mejor evidencia disponible. En este marco parece apropiado incluir la dieta combinada como una alternativa más entre las recomendadas para el tratamiento de la HTA estadío 1 y para su posible prevención en pacientes sanos. Además ambas dietas con un mayor porcentaje de fibras, un menor porcentaje de grasas tienen también otras ventajas adicionales sobre la salud.

Dr. José Ustarán Medicina Familiar C.E.M.I.C. 